\title{
Gene Expression Pattern in Human Monocytes as a Surrogate Marker for Systemic Inflammatory Response Syndrome (SIRS)
}

\author{
Grit Wiegand, ${ }^{1}$ Kathleen Selleng, ${ }^{1}$ Matthias Gründling, ${ }^{2}$ and \\ Robert S. Jack ${ }^{1}$ \\ ${ }^{1}$ Institut für Immunologie und Transfusionsmedizin, Klinikum der \\ Universität Greifswald, Greifswald, Germany \\ ${ }^{2}$ Klinik für Anästhesiologie und Intensivmedizin, Greifswald, Germany \\ Accepted January 5, 1999.
}

\begin{abstract}
Background: Systemic inflammatory response syndrome (SIRS) is a mild inflammmatory episode which, in a minority of patients, may deteriorate into septic shock. In the mouse, injection of bacteria or bacterial endotoxin induces systemic inflammation through the activation of blood monocytes, which leads to lethal shock. A number of intervention strategies have been shown to prevent progression to shock in mouse model systems. However, recent clinical trials of a number of these therapeutic strategies in patients have been uniformly disappointing. In contrast to the situation in the mouse models, there may be many different ways to initiate systemic inflammmation in patients and not all of them need necessarily involve activation of blood monocytes. If there is no unifying mechanism behind the induction of systemic inflammation in patients and no common rules governing its development, then it is unlikely that generally
\end{abstract}

applicable therapeutic strategies will be found that can prevent progression into shock.

Materials and Methods: We used differential display to compare gene expression patterns in monocytes of recentadmission multi-trauma patients with clinically diagnosed SIRS to the patterns in monocytes of healthy controls.

Results: Of seven differentially displayed bands that were recovered and sequenced, five were associated with SIRS and two were preferentially expressed in the monocytes of healthy controls.

Conclusion: The data show that monocytes of SIRS patients are in an activation state that is different from that of monocytes from the healthy controls, that monocytes from many individual patients share similar patterns of differentially expressed sequences, and that by this criterion, the multi-trauma SIRS patients are a remarkably coherent group.

\section{Introduction}

Systemic inflammatory response syndrome (SIRS) ( 1 ) is a mild systemic inflammation that is usually transitory. However, in about $10 \%$ of cases in intensive care units, the clinical picture deteriorates and these patients may die of septic shock (2). In contrast to SIRS, septic shock is a syndrome with high mortality for which no

Address correspondence and reprint requests to: Dr. Robert S. Jack, Institut für Immunologie und Transfusionsmedizin, Klinikum der Universität Greifswald, Sauerbruchstrasse, D-17489 Greifswald, Germany. Phone: (49) 383486 5455; Fax: (49) 3834865455 causal therapy is currently available. In animal models the induction of septic shock has been shown to involve a complex cascade of events that includes dysregulation of the cytokine network. A number of intervention strategies have been devised that aim to interfere with the initiation or progression of the inflammatory cascade. Administration of soluble forms of the TNF- $\alpha$ receptor (3), elimination of IL-1 $(4,5)$, or treatment with anti-LPS antibodies (6), have all been shown to block induction of lethal shock in mice. Recent attempts to use these agents for the treatment of patients however, have been uni- 
formly disappointing (6-8). The reason why strategies that are successful in animal models remain ineffective in patients is unclear.

One obvious difference between the two situations is that in the mouse models an inflammatory cascade is initiated in inbred animals with a defined pyrogen-usually lipopolysaccharide (LPS). This results in the activation of monocytes and macrophages that release the proinflammatory mediators that induce a defined inflammatory cascade (9). An experimentally induced cascade of this kind can then be interrupted by application of the appropriate countermediator. In contrast, in patients the cause or causes of the systemic inflammation are not known in detail and the nature of the inflammatory cascade induced is unclear. Since SIRS is operationally defined on the basis of clinical data, it identifies patients with a particular spectrum of symptoms without necessarily implying any common underlying pathophysiological mechanisms. It remains controversial whether SIRS defines a coherent patient group who all suffer from a systemic inflammation with similar properties or whether it is merely a catch-all term for a broad array of distinct physiological states, all of which satisfy the clinical criteria of SIRS (10). To distinguish these two possibilities, we used differential display (11) to ask first whether monocytes from multi-trauma patients satisfying the Consensus Conference definition of SIRS (1) are in a different activation state from that of the monocytes of healthy controls and second, whether the monocytes from individual SIRS patients share particular differentially expressed genes that would imply a similar activation status.

\section{Materials and Methods}

SIRS Criteria

The criteria used to define SIRS in this study were those layed out by the American College of Chest Physicians/Society of Critical Care Medicine Consensus Conference for sepsis and organ failure (1). Inclusion criteria were informed consent, age $>17$ years, recent-admission multitrauma, and a diagnosis of SIRS. Exclusion criteria were lack of informed consent, known immunosupressive therapy, cancer, or AIDS. SIRS is defined as a situation in which an acute alteration from baseline results in two or more of the following criteria being met: (1) temperature $>38^{\circ} \mathrm{C}$ or $<36^{\circ} \mathrm{C}$; (2) heart rate $>90$ beats $/ \mathrm{min}$;
(3) respiratory rate $>20$ breaths/min or $\mathrm{PaCO}_{2}$ $<32 \mathrm{mmHg}$; (4) white blood cell count $>12,000 / \mathrm{ml}$ or $<4000 / \mathrm{ml}$ or $>10 \%$ immature neutrophils.

\section{Patients}

Blood samples were taken from SIRS patients in the intensive care unit of the Department of Anesthesia and from healthy volunteers. Twenty-six monocyte preparations were made from a total of 17 SIRS patients. Each individual patient was identified by a letter (A-Q). All suffered from multi-trauma as a result of accidents. The mean age was 41.6 years (range 17-61) and the mean APACHE II score on admission was 24.2 (range 15-34). All patients or their relatives gave informed consent and the study was approved by the local medical ethics committee. The timing of sample withdrawal relative to the start of SIRS is shown in Table 1. In the cases of patients C, D, F, $\mathrm{H}, \mathrm{L}$, and $\mathrm{O}$, a second sample was taken. In the case of patient E, three further blood samples were taken. All of these patients remained in SIRS throughout the study period.

\section{Monocyte Preparation}

Monocytes were isolated from whole blood in a two-step procedure. Briefly, mononuclear cells were recovered from $10-\mathrm{ml}$ samples of heparinized whole blood by Ficoll step-gradient (density 1.077, Seromed, Berlin) centrifugation using LeucoSep tubes (Greiner, Solingen, Germany). The cells at the Ficoll interface were recovered and all further steps were carried out at $+4^{\circ} \mathrm{C}$ in the presence of $0.1 \% \mathrm{NaN}_{3}$ to minimize the risk of artefactual activation. The cells were washed three times in ice-cold phosphate-buffered saline (PBS) $/ \mathrm{NaN}_{3}$ and resuspended in $280 \mu \mathrm{l}$ cold PBS/ $\mathrm{NaN}_{3}$ supplemented with $0.5 \%$ bovine serum albumin (BSA). The suspension was briefly vortexed to disperse cell clumps and was incubated at $4^{\circ} \mathrm{C}$ for $20 \mathrm{~min}$ with $70 \mu \mathrm{l}$ of $\mathrm{CD} 14$ magnetic beads (Miltenyi Biotec, Bergisch Gladbach, Germany). The cells were loaded onto a MiniMACS magnetic separation column. After washing the retained cells were eluted from the column in $\mathrm{PBS} / \mathrm{NaN}_{3} / \mathrm{BSA}$, vortexed briefly, and rerun on the same column a second time. Cells recovered from the second run on the column were characterized by FACScan analysis. 
Table 1. Patient data

\begin{tabular}{|c|c|c|c|c|c|}
\hline Patient & $\begin{array}{c}\text { Age } \\
\text { (years) }\end{array}$ & Sex & $\begin{array}{l}\text { APACHE } \\
\text { Score }^{a}\end{array}$ & Outcome & $\begin{array}{c}\text { Samples Taken } \\
\left.\text { (days }^{b}\right)\end{array}$ \\
\hline A & 60 & $M$ & 34 & Died & 5 \\
\hline B & 53 & $\mathrm{~F}$ & 25 & Died & 6 \\
\hline C & 18 & $M$ & 15 & Recovered & 2,6 \\
\hline $\mathrm{D}$ & 34 & M & 15 & Recovered & 18,23 \\
\hline E & 55 & M & 26 & Recovered & $4,5,11,17$ \\
\hline $\mathrm{F}$ & 54 & $M$ & 26 & Recovered & 1,3 \\
\hline G & 29 & M & 29 & Recovered & 2 \\
\hline $\mathrm{H}$ & 56 & M & 23 & Recovered & 1,2 \\
\hline I & 55 & M & 32 & Died & 1 \\
\hline $\mathrm{J}$ & 48 & $M$ & 32 & Died & 9 \\
\hline $\mathrm{K}$ & 20 & M & 22 & Recovered & 6 \\
\hline $\mathrm{L}$ & 51 & $M$ & 23 & Recovered & 2,5 \\
\hline M & 37 & $M$ & 21 & Recovered & 2 \\
\hline $\mathrm{N}$ & 61 & $M$ & 16 & Recovered & 4 \\
\hline $\mathrm{O}$ & 34 & $M$ & 28 & Recovered & 2,4 \\
\hline$P$ & 17 & $\mathrm{~F}$ & 23 & Recovered & 3 \\
\hline $\mathrm{Q}$ & 25 & $M$ & 22 & Died & 5 \\
\hline
\end{tabular}

${ }^{a}$ APACHE score determined on admission.

${ }^{b}$ Time point at which SIRS was diagnosed is defined as day 0 . Second and subsequent samples were taken from patients who remained in SIRS over the entire time period.

\section{FACScan Analysis of Monocytes}

To assess the purity of the isolated monocytes a sample was stained with phycoerythrin-labeled antibody to CD45 (Becton Dickinson), which labeled all of the leukocytes in the sample. Simultaneously, the cells were stained with FITC-labelled antibody to the monocyte marker CDl4 (Becton Dickinson). We determined the final purity by analyzing the stained cell sample in a FACScan. Cells were judged to be monocytes if they were $\mathrm{CD} 45^{\mathrm{hi}}$ and $\mathrm{CD} 14^{\mathrm{hi}}$ and had the appropriate forward- and side-scatter properties (12). Preparations containing $<97 \%$ monocytes were rejected. Overall yields of monocytes were typically $50 \%$. Monocyte-specific alpha naphthol esterase was visualized on smears of the cell preparations (13).

\section{Preparation of RNA and $c D N A$}

Total RNA was prepared from monocytes lysed in guanidinium isothiocyanat using the Micro RNA isolation kit (Stratagene, Heidelberg). Total RNA from around $5 \times 10^{5}$ cells (average yield
$0.75 \mu \mathrm{g})$ was treated with 10 units of RNase-free DNase 1. RNA preparations were routinely checked for contamination with genomic DNA by polymerase chain reaction (PCR) using either the differential display oligonucleotides or primers specific for the second exon of the CD14 gene. In no case was DNA contamination of the RNA preparations detected.

The RNA preparations were reverse transcribed in a final volume of $20 \mu$ l containing 20 $\mu \mathrm{M}$ of each dNTP, $10 \mu \mathrm{M}$ DTT, $2.5 \mu \mathrm{M}$ of one of the four oligonucleotide sets, and first-strand buffer (Gibco-BRL). The reaction mix was heated to $65^{\circ} \mathrm{C}$ for $5 \mathrm{~min}$ then cooled to $37^{\circ} \mathrm{C}$ for $10 \mathrm{~min}$, and 200 units of reverse transcriptase (Superscript plus, Gibco-BRL) was added. After 50 min at $37^{\circ} \mathrm{C}$ the reaction was terminated by heating to $95^{\circ} \mathrm{C}$ for $5 \mathrm{~min}$. The oligo-sets used were:

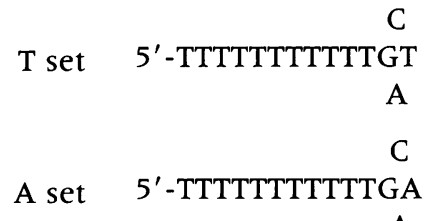




$\begin{array}{cr}\text { G set } & \text { C } \\ & \text { 5'-TTTTTTTTTTTGG } \\ \text { C set } & 5 \text {-TTTTTTTTTTTC } \\ \text { C } \\ \text { A }\end{array}$

Semi-quantitative PCR

cDNA preparations were normalized for their $\beta$-actin content by titration against a fixed amount of an internal standard in a semi-quantitative PCR (14). The internal standard yielded a fragment of 438 bp which was readily separated on $1.5 \%$ agarose gels from the $719 \mathrm{bp}$ product derived from the $\beta$-actin cDNA.

\section{Differential Display}

One microliter of the appropriate dilution of the cDNA preparation was taken for PCR amplification. Each PCR reaction $(20 \mu \mathrm{l})$ contained 2.5 $\mu \mathrm{M}$ of one member of the appropriate oligo-dT set ( $3^{\prime}$ primer), $0.5 \mu \mathrm{M}$ of one of the 26 differential display decamers ( $5^{\prime}$ primer), $5 \mu \mathrm{M}$ of each dNTP, Taq polymerase buffer (Pharmacia), 6.25 $\mu \mathrm{Ci}$ of $\alpha-\left[{ }^{35} \mathrm{~S}\right] \mathrm{dATP}, 1$ unit of Taq polymerase (Pharmacia), and anti-Taq polymerase antibody (Clontech). Amplification was carried out for $\mathbf{4 0}$ cycles of $94^{\circ} \mathrm{C}$ for $30 \mathrm{sec}, 40^{\circ} \mathrm{C}$ for $2 \mathrm{~min}, 72^{\circ} \mathrm{C}$ for $30 \mathrm{sec}$, and a final extension of $5 \mathrm{~min}$ at $72^{\circ} \mathrm{C}$. The differential display decamers used were those suggested by Bauer et al. (15). Fragments generated were fractionated on standard $6 \%$ sequencing gels and visualized by autoradiography.

\section{Cloning of Differentially Expressed Fragments}

Fragments were excised from differential display gels and reamplified with the same primers, and the PCR products were cloned into the pT-Adv vector (Clontech, Heidelberg). In the case of band 12 , which is present as a doublet, only the upper band was excised. Each differentially expressed band was recovered from three individuals and clones from each were sequenced using a ThermoSequenase cycle sequencing kit (Amersham Buchler, Braunschweig, Germany). Sequence reactions were analyzed on a LI-COR DNA Sequencer (MWG-Biotech, Ebersberg, Germany).

\section{Results \\ Patients}

The age, sex, and APACHE scores on admission of the patients involved in this study are shown in Table 1, as are the time points of blood sample withdrawal. These polytrauma patients were all recent admission accident victims so that effects arising from chronic illness are minimised.

\section{Preparation of Monocytes}

Differential display analyses are crucially dependent on the purity of the cell populations used. If one of the cell populations being compared is contaminated then differences of gene expression in the two populations may be due simply to the different degrees of contamination. To isolate monocytes, we make use of the fact that in human blood, the cell surface antigen CD14 is expressed in high amounts on monocytes and in much lower amounts on granulocytes, and is not detectable on other cells. We first remove erythrocytes and granulocytes by Ficoll-gradient centrifugation. Monocytes are then recovered from the pool of mononuclear cells by two rounds of cell sorting on magnetic beads coated with an anti-CD 14 monoclonal antibody. The monocytes recovered by this procedure have a purity estimated by FACS analysis of $97 \%$ (Fig. 1). In this isolation procedure the expression of $\mathrm{CDI} 4$ is exploited both for the magnetic cell sorting and for the analysis of monocyte purity. It is therefore important to ensure that the systemic inflammation does not lead to CD14 expression on blood cells other than monocytes. We have controlled for this point by examining the purity of the monocytes, using as an independent marker the expression of the monocyte-specific enzyme $\alpha$-naphthol esterase. Samples of isolated monocytes were prepared both from controls and from SIRS patients and stained for $\alpha$-naphthol esterase expression. For this analysis 300 cells per slide were examined. In monocyte samples in which the FACScan analysis showed 97\% monocytes, we routinely found $98-99 \% \alpha$-naphthol esterase-positive cells. The slight discrepancy between the assays may simply be due to the inherently greater accuracy of the FACScan procedure in which a much larger number of cells are quantitated.

This result assured us that the cells we examined from the SIRS and control groups were indeed monocytes. We cannot, of course, 

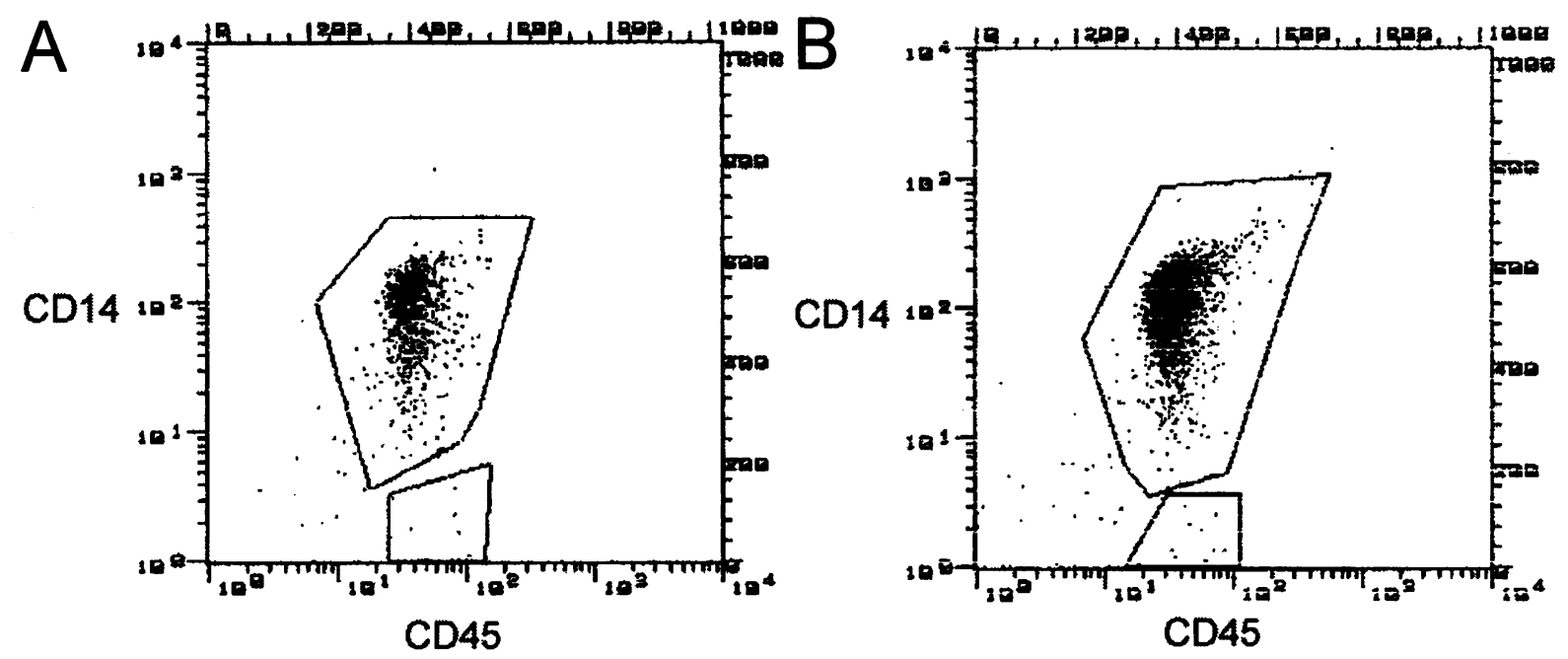

Fig. 1. FACS analysis of isolated monocytes from a SIRS patient (A) and from a healthy control (B). Monocyte preparations were labeled with FITC-anti-CD45 and PE-anti-CD14. CD45 expression is plotted against CD14 expression.

exclude the possibility that in SIRS patients a population of monocytes became CD14 negative and thus escaped our analysis.

\section{Selection of Oligonucleotide Pairs}

In the original description of the differential display procedure, a set of 312 oligonucleotide pairs were defined that on statistical grounds were thought to be able to detect essentially all mRNA species (15). The small amount of cDNA recovered from the necessarily limited volumes of blood available from patients was insufficient to carry out a complete analysis with all 312 differential display primer combinations. For this reason, we first determined which oligonucleotide pairs yielded the most complex and hence most informative patterns using cDNA prepared from monocytes of a healthy individual. Part of this analysis is shown in Figure 2. In this experiment, the anchor oligonucleotide $\mathrm{T}_{11} \mathrm{CA}$ was tested with each of the 26 oligonucleotide decamers. Some combinations yielded no detectable products (e.g., lanes 4, 11, 16, and 17). Others such as those shown in lanes $2,3,5,7,10,12,15,18$, 20, 21, and 25 yielded patterns composed of only a few bands. Usefully complex patterns were generated by only a minority of the primer pairs (e.g., lanes 1, 14, 24, and 26). This experiment was repeated and similar gels were run for each of the other 11 anchor oligonucleotides. Out of these 312 tested primer com- binations, those shown in Table 2 were selected for further study since they yielded the most complex banding patterns. This approach has the advantage of maximizing the information we can extract from the limited amount of patient cDNA available. It does suffer, however, from the limitation of missing SIRS-specific transcripts detectable only with a primer pair that yields no signal with cDNA from healthy controls.

\section{Differential Display}

Because the yield of mRNA recovered from the isolated monocytes may vary from individual to individual, we normalized the amount of cDNA in the preparations using the $ß$-actin mRNA content as standard. The preselected primer pairs were then used to carry out a comparison between cDNA preparations from monocytes of SIRS patients and those from healthy controls. Since the banding patterns obtained were complex, we simplified the comparisons by analyzing multiple SIRS and multiple control samples on a single gel.

A typical differential display experiment using anchor primer $5^{\prime}-\mathrm{T}_{11} \mathrm{CA}-3^{\prime}$ and decamer $5^{\prime}$-GATCTAAGGC- ${ }^{\prime}$ is shown in Figure 3. This is the combination of the anchor primer and decamer 26 that is shown in Figure 2. PCR products from 23 SIRS monocyte preparations and from 19 healthy controls were analyzed on the same gel. Each patient is represented by a 


\section{decamer}
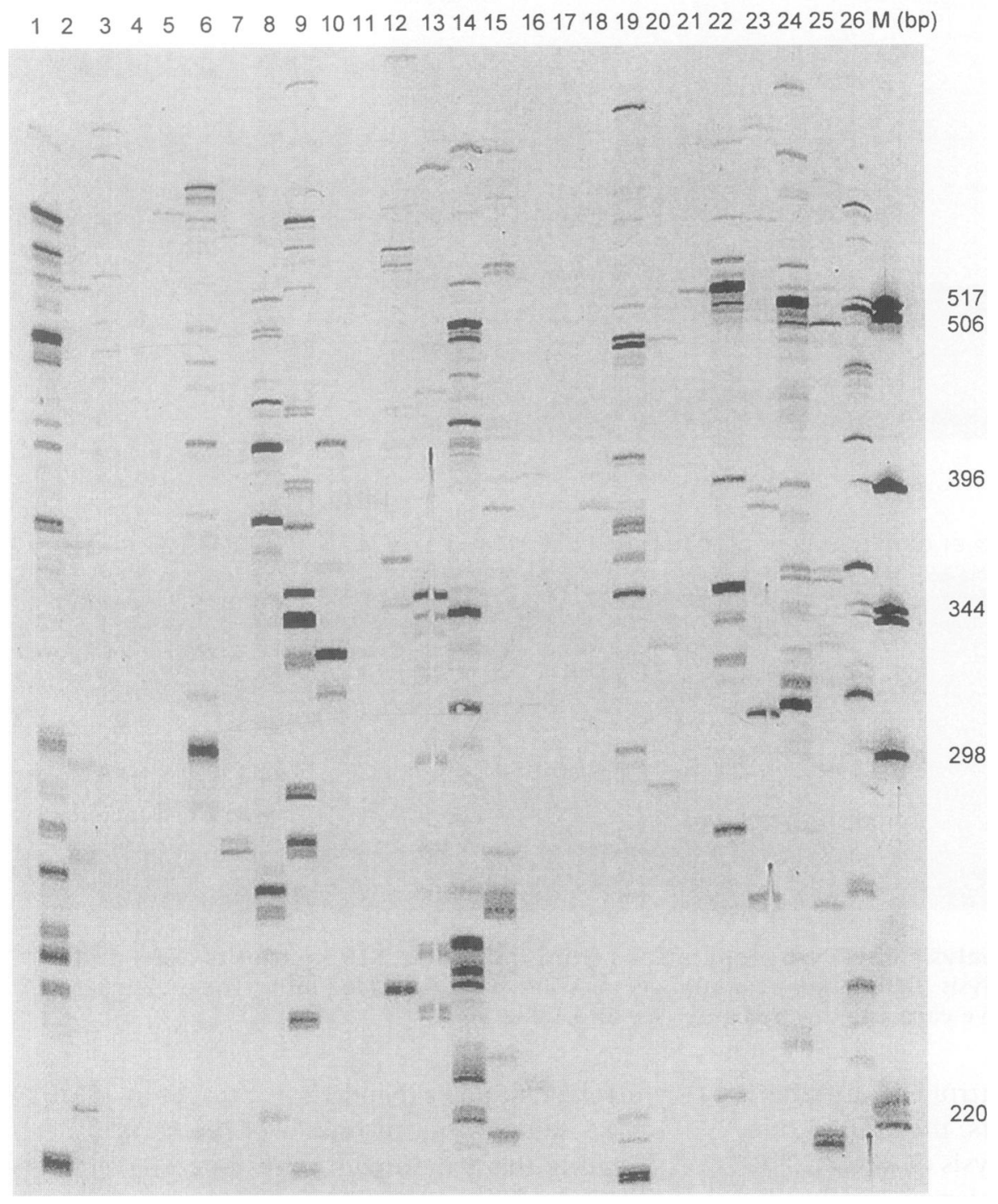

Fig. 2. Selection of oligonucleotide pairs for use in differential display. A cDNA preparation from a healthy individual was used for PCR with the anchored oligo $5^{\prime}-T_{(11)} C A$ and all 26 of the decamers suggested by Bauer et al. (15). Products were fractionated on a $6 \%$ sequencing gel.

Table 2. Bands differentially expressed in monocyte preparations from SIRS patients and normal controls

\begin{tabular}{lccrrl}
\hline Band & Anchor Primer & Decamer Primer & SIRS & Controls & $\boldsymbol{p}^{\boldsymbol{a}}$ \\
\hline 2 & T(11)GC & 5'-GATCATAGCG & $3 / 20$ & $14 / 20$ & $<0.0005$ \\
3 & T(11)GC & 5'-GATCATAGCG & $2 / 20$ & $13 / 20$ & $<0.0005$ \\
4 & T(11)AA & 5'-GATCTGACAC & $10 / 25$ & $0 / 19$ & $<0.005$ \\
10 & T(1) CA & 5'-GATCATCGTC & $22 / 23$ & $2 / 19$ & $<0.0005$ \\
12 & T(11)CA & 5'-GATCTAAGGC & $19 / 23$ & $3 / 19$ & $<0.0005$ \\
15 & T(11)AG & 5'-TCGATACAGG & $19 / 24$ & $7 / 20$ & $<0.005$ \\
16 & T(11)AG & 5'-CTGCTTGATG & $17 / 24$ & $6 / 20$ & $<0.01$ \\
\hline
\end{tabular}

${ }^{a} p$ values were determined by the chi-squared test. 


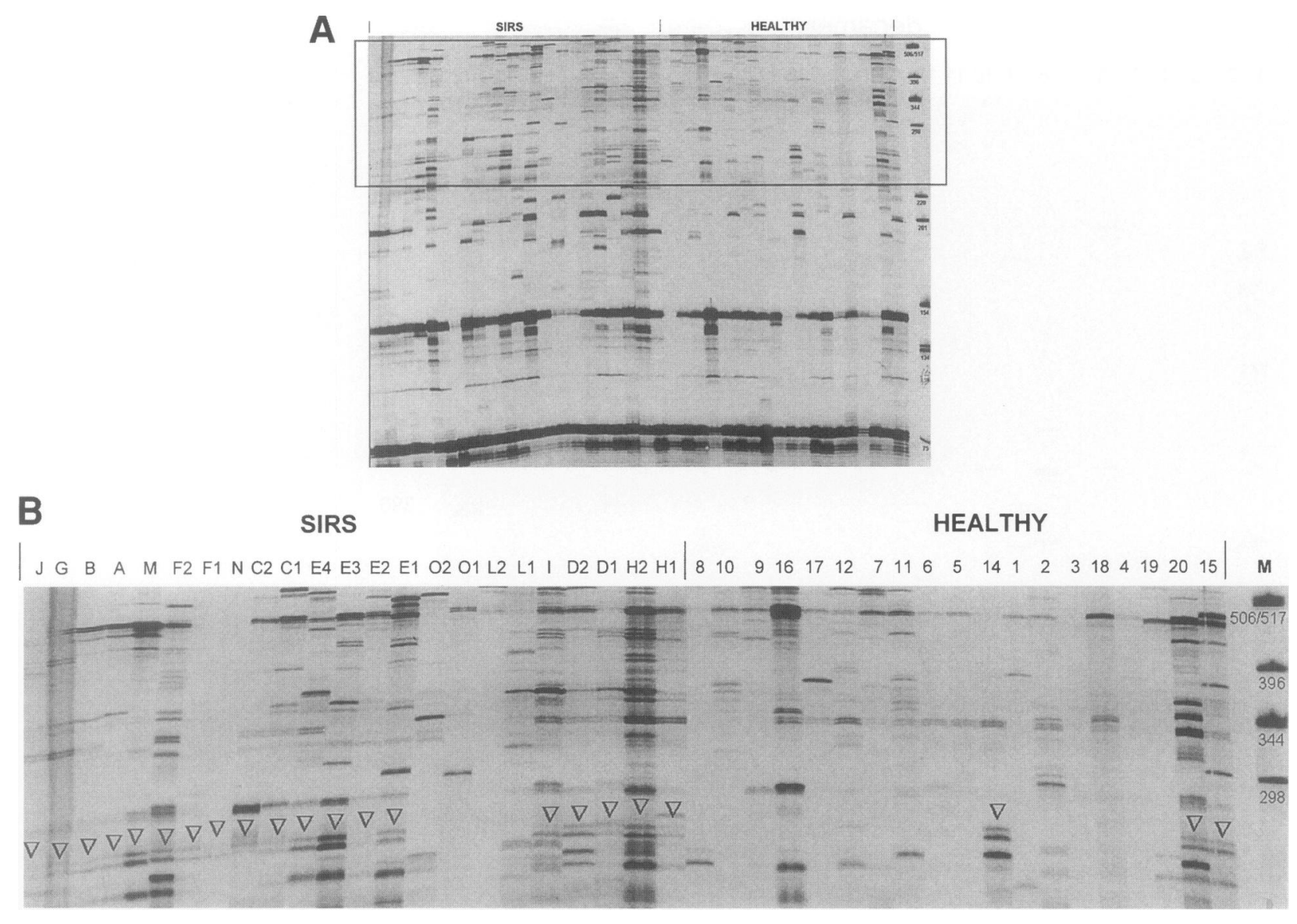

Fig. 3. Differential display analysis of cDNA preparations from individual SIRS patients (left) and controls (right). (A) Entire analysis. (B) Marked region of gel in A shown in expanded form. The individual patient designations are shown above each lane in the figure (see also Table 1).

letter and each individual control by a number. For seven of the SIRS patients, more than one sample was available for analysis (Table 2 ). The elements of the pattern recovered can be divided into three groups. The first consists of bands present in a single sample or in a few samples but whose expression does not depend on whether the source of the cDNA was SIRS or normal monocytes. The second group consists of bands present in all or nearly all samples, again irrespective of whether the monocytes came from SIRS patients or controls. Most of the bands fell into these groups and were of no further interest for analysis. The third group consists of those bands present mainly in the cDNA samples from the monocytes of the SIRS patients or mainly in the cDNA from the control monocytes.

\section{Bands Preferentially Expressed in SIRS Monocytes}

In the upper part of the gel in Figure 3A, which is shown in expanded form in Figure $3 B$, the arrowed doublet (band 12) is present in 19 of 23 SIRS samples but in only 3 of the controls. Repeating this experiment with different oligonucleotide pairs allowed us to examine different parts of the cDNA population. Similar gels were run for each of the oligonucleotide combinations shown in Table 2. These seven oligonucleotide pairs detected a total of seven bands whose expression in monocytes from SIRS patients was significantly different (chi-squared test, see Table 2) from their expression in monocytes of controls. The expression of these bands in the SIRS patients and in the controls is shown in Tables 3 and 4 . Band 10 was expressed in all but one of the SIRS monocyte preparations tested. All but two of the SIRS patients expressing band 10 simultaneously expressed band 12 . In contrast, only two of the tested controls expressed band 10 and neither of them simultaneously expressed band 12. The expression of bands 4, 15, and 16 was also correlated with SIRS (Tables 2 and 3). 
Table 3. Expression of bands in monocytes of SIRS patients

\begin{tabular}{|c|c|c|c|c|c|c|c|c|c|c|c|c|c|c|c|c|c|c|c|c|c|c|c|c|c|c|}
\hline Band & $\mathbf{A}$ & B & Cl & C2 & DI & D2 & El & E2 & E3 & E4 & F1 & F2 & $\mathbf{G}$ & HI & H2 & I & $\mathbf{J}$ & $\mathbf{K}$ & $\mathbf{L l}$ & $\mathbf{L} 2$ & $\mathbf{M}$ & $\mathbf{N}$ & 01 & 02 & $\mathbf{P}$ & $\mathbf{Q}$ \\
\hline 2 & - & - & nd & - & - & - & - & - & nd & nd & + & - & nd & - & - & . & - & + & - & - & - & - & - & nd & + & nd \\
\hline 3 & - & - & nd & - & - & - & - & - & nd & nd & - & - & nd & - & - & + & - & + & - & - & - & - & - & nd & - & nd \\
\hline 4 & + & + & + & - & + & - & + & nd & + & + & - & + & - & - & - & - & + & + & - & - & - & - & - & - & - & - \\
\hline 16 & + & + & + & - & + & + & + & nd & + & + & + & + & + & + & + & + & - & + & + & - & - & - & + & - & nd & - \\
\hline 15 & + & + & + & + & + & + & + & nd & + & - & + & + & + & + & + & + & + & + & - & + & + & + & - & - & nd & - \\
\hline 12 & + & + & + & + & + & + & + & + & + & + & + & + & + & + & + & $T$ & + & nd & - & - & $T$ & + & - & - & ad & nd \\
\hline 10 & + & + & + & + & + & + & + & + & + & + & + & + & + & + & + & + & + & $\mathrm{n}$ & + & - & + & + & 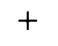 & + & nd & \\
\hline
\end{tabular}

nd, not determined.

Table 4. Expression of bands in monocytes of controls

\begin{tabular}{llllllllllllllllllllll}
\hline Band & 1 & 2 & 3 & 4 & 5 & 6 & 7 & 8 & 9 & 10 & 11 & 12 & 13 & 14 & 15 & 16 & 17 & 18 & 19 & 20 \\
\hline 2 & - & - & - & - & + & + & + & + & + & + & + & + & + & + & + & + & + & - & + & - \\
3 & - & - & - & - & + & + & + & + & + & + & + & + & + & + & + & - & - & + & - & + \\
4 & - & - & - & - & - & - & - & - & - & - & - & - & nd & - & - & - & - & - & - & - \\
16 & - & - & - & - & - & - & - & - & - & + & - & + & - & - & + & + & + & - & + & - \\
15 & - & - & - & - & - & + & - & - & - & + & - & - & - & - & + & + & - & + & + & + \\
12 & - & - & - & - & - & - & - & - & - & - & - & - & nd & + & + & - & - & - & - & + \\
10 & - & - & + & - & - & - & - & - & - & - & - & - & nd & - & - & - & - & + & - & -
\end{tabular}

nd, not determined.

\section{Bands Preferentially Expressed in Control Monocytes}

The expression of bands 2 and 3 was negatively correlated with SIRS. Band 2, which was detected using the oligonucleotides $5^{\prime}-\mathrm{T}_{11} \mathrm{GC}-3^{\prime}$ and $5^{\prime}$-GATCATAGCG-3', was expressed in 14 of 20 cDNA preparations from monocytes of controls but in only 3 out of 20 cDNA preparations from SIRS patients. A similar result was found for band 3, detected using the same oligonucleotides, which was expressed in 13 of 20 controls but in only 2 out of 20 SIRS monocyte preparations (Table 4). Though 11 of the 20 controls expressed both of these elements simultaneously, only one of the SIRS patients (Patient K) did so.

\section{Stability of the Expression Pattern}

Two blood samples were withdrawn from SIRS patients $\mathrm{C}, \mathrm{D}, \mathrm{F}, \mathrm{H}, \mathrm{L}$, and $\mathrm{O}$ and four samples were withdrawn from Patient $\mathrm{E}$ (Table 1). In the case of band 15, one patient (E) changed from being initially positive to being negative in the last sample. Band 16 was initially positive in patients $\mathrm{C}, \mathrm{L}$, and $\mathrm{O}$ and then became negative. Band 4 also changed its expression pattern. It was initially expressed in patients $C$ and $D$ but was no longer detectable at the second time point, whereas in patient $\mathrm{F}$ its expression was seen only at the second time point. In contrast, the expression patterns of the SIRS-associated elements 10 and 12 were robust in the sense that within each sample they remained unaltered over the time points examined.

\section{Recovery of Differentially Expressed Sequences}

The bands 2, 3, 4, 10, and 12 were recovered from $6 \%$ acrylamide gels and ligated into the Clontech dAT vector. For each, band clones were 


\author{
Band 2 \\ GATCATAGCG GTGTCGCAGA GTAGGAATTT TGTTTTGGTG AGCAGTGGAA \\ GCTAGGCTGC TTAGGGTAAT AGAGGGCCAG CGGAGGTGCA GGCTGAGAGG \\ TCAACTGAAA AGTTTAATGT GACAGGCAAA AAAAAAAA
}

\author{
Band 3 \\ GATCATAGCG GGAAGGGCTC CATTTCCCAG GGAAAATAAC TATCATGCAT \\ CTTTCTCTGT AAATACAAGT TTGTAAACAC TGGCTAATGA CAGAGGGATG \\ GAGAGATATG TGATAAAGCA AGTAAAATGT AAATGGTAGA AACTAAGTGG \\ TGGTATGTGG GTGCTCACTT AACACTCCTT CAACTTATCT GTATGTTAGA \\ AAAATTTCAT AAGAAAATA
}

Band 4

GATCTGACAC AGTAATATGT TTCAGGCTTA GTCTATACTT TCTCTACCAC AGACACCCCA TCCTATCCCA TCATAGTCTA AGTTTCAATC AGCATTCCCT TAACTATAAA TGTCTCATTT TACCTGGGAG AGGTCAATGC CCCAAGGGCA CTGTGAGTTA AGAAACTGGT CAAGAATCAG CAATCCCTTG TAGGTGTCAG ATC

Band 10

ACCACAGTCC ATGCCATCAC CATCATCACC ACCATCACCA TCATCAGCAG CAGCAGCATC ACCACCAGCA CCATCATCAT TATTTCATCA CCACCACCAT TGGCATCACC ATCAGGACCA TCATCATCAC TGACCATCAT CATCATCACA TGACCATGAT CATCACTGAC CATCATCATC ATCACATGAC CATGATC

Band 12

GATCTAAGGC CCAATAACAA GTTTATCTCT TTCCCACGAT TAAATAGGTG TTTATAAATC TTGTGGCTGG TTTATTGTTG AAAAATTATA AGCTAGTGTC TAGTATCATT GTAGTAGATT TTTTCCACAA GATTTCCTTG AAACTGTTTA ATACAACAGT TTCAGGTGCT TAGTGTACTT ATTTGTTCAT GAAAAAAAAA AA

recovered from at least three individuals and sequenced. The sequences of these elements are shown in Figure 4. A BLAST analysis of these sequences against the NCBI database showed that they do not correspond to currently identified genes.

\section{Discussion}

Differentially Displayed Bands

In the differential display procedure, PCR amplification employs short oligonucleotides and low annealing temperatures, and these factors may lead to erroneous priming. This may explain the origin of many of the bands present in only one or a few of the 44 independently prepared RNA samples examined. Whether such bands are truly differentially expressed or are merely the result of priming artefacts must be tested by some other means, such as northern analysis. In contrast, bands that appear in monocytes of many individuals are unlikely to be due to ran-
Fig. 4. Sequences of cloned bands. Anchor and decamer oligonucleotides are shown in bold type. Bands 2 and 12 contain one anchor and one decamer sequence. Band 4 contains two copies of the decamer in opposite orientation. A single copy of the appropriate decamer is found in bands 3 and 10 . dom error in the amplification, particularly when their expression is largely restricted to either the SIRS or the control group. Thus band 2, which was found in 14 out of 20 controls but in only 3 out of 20 monocyte preparations from SIRS patients, and band 10, which was present in 22 of 23 cDNA preparations from SIRS patients but in only 2 out of the 19 tested controls, are unlikely to be due to priming artefacts.

Differentially Displayed Bands and the Nature of SIRS

It is as still unclear what the Consensus Conference definition of SIRS actually describes. Two extreme possibilities are shown in Figure 5. The first is that SIRS defines a relatively coherent patient group (Fig. 5A). In this model, the diagnosis of SIRS reflects a common underlying pathophysiological mechanism in all of these patients. The second possibility is that SIRS describes a wide array of different pathophysiological states (Fig. 5B). These different states may 


\section{A}

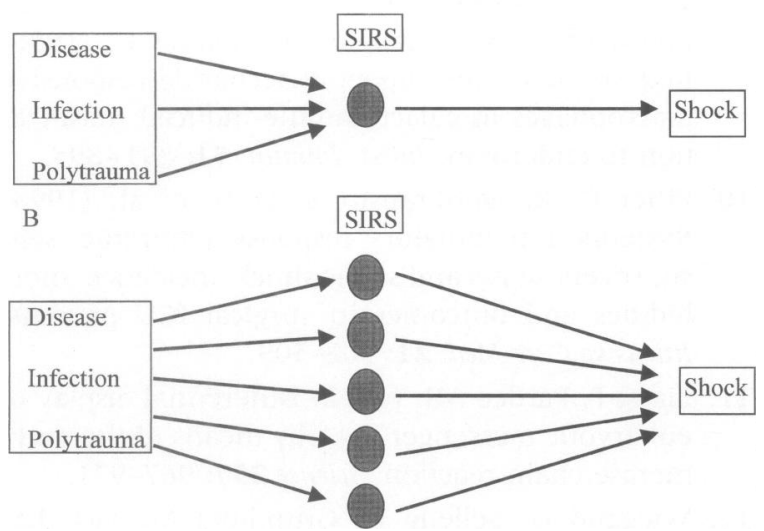

Fig. 5. Two models for induction of SIRS and progression to septic shock. (A) The SIRS definition describes a coherent pathophysiological state that may progress into shock. (B) SIRS describes a range of pathophysiological states that need not be mechanistically related. Each of these states may then progress to shock by a different route.

then deteriorate to shock by mechanistically unrelated routes. If this latter possibility is correct and there is no unifying mechanism underlying the induction of SIRS and the transition to shock, then it is unlikely that generally applicable treatment strategies to block this transition will be found.

Using the preselected oligonucleotide pairs, we found a total of seven bands whose expression in monocytes correlates with their origin in SIRS patients or controls. None of the five bands associated with SIRS was present in all SIRS patients and absent from all of the controls. However, 22 of 23 SIRS monocyte preparations expressed band 10, whereas only 2 out of 19 tested controls did so. Out of 22 SIRS monocyte preparations 17 simultaneously expressed bands 10 , 12 , and 15 whereas none of the controls did so $(p<0.0005)$. On the other hand the simultaneous expression of bands 2 and 3 is negatively correlated with SIRS $(p<0.001)$. These differences in the pattern of RNA expression support the notion that in SIRS patients the monocytes are in a different activation state than that of monocytes from controls. Given the rather general nature of the clinical definition of SIRS, the correlations are surprisingly good. The existence of such shared patterns of gene expression favors the model shown in Figure 5A and argues that by these criteria, the multi-trauma patients examined are a remarkably coherent group.

\section{Differentially Expressed Sequences}

Monocytes activated in vitro with LPS and $\gamma$-interferon transcriptionally up-regulate a battery of proinflammatory cytokines and may perhaps provide a model of the activation status of monocytes in shock patients. However, SIRS is in contrast a mild inflammatory condition in which the degree of monocyte activation is still under control. Progression from SIRS to shock may be accompanied by a series of small changes in the pattern of gene expression in monocytes which results in a progressive loss of control over the activation process. Our results fit this model. None of the differentially displayed sequences appears to be a major transcriptional product and none of the recovered sequences codes for a known proinflammatory mediator. In the experiment shown in Figure 3, band 12 is not an intense component of the displayed pattern. This is also true for bands $2,3,4,10,15$, and 16, which were detected on gels run from experiments carried out with the other oligonucleotide pairs tested. While the differential display procedure is by no means quantitative, it would nevertheless seem unlikely that any of the identified bands represents a massively induced gene.

Only a small fraction of the genes in the human genome have been identified thus far. Indeed, it has recently been estimated that $<50 \%$ of human genes are currently even represented in the Unigene public EST database (16). Thus it is not surprising that our differentially expressed sequences do not correlate with known genes. We are currently attempting to isolate full-length cDNAs of these clones so as to identify the gene products.

It will be of interest to examine a larger panel of SIRS and shock patients. We aim to determine whether the pattern of expression or degree of transcription of these genes might be used to subdivide SIRS patients into different risk groups. For this purpose, quantitative PCR using specific oligonucleotides prepared from the sequenced fragments will permit a more detailed analysis than can be achieved with differential display.

\section{Acknowledgments}

This work was supporterd by the DFG through grant IIB6 Ja 729 /1-2. 


\section{References}

1. American College of Chest Physicians, and Society of Critical Care Medicine Consensus Conference. (1992) Definitions for sepsis and organ failure and guidelines for the use of innovative therapies in sepsis. Crit. Care Med. 20: 864-874.

2. Bone RC, Fischer CJ, Clemmer TP, Slotman GJ, Metz CA, Balk RA. (1989) Sepsis syndrome: a valid clinical entity. Crit. Care Med. 17: 389-393.

3. Rogy MA, Auffenberg T, Espar NJ, et al. (1995) Human tumor necrosis factor receptor (p55) and interleukin 10 gene transfer in the mouse reduces mortality to lethal endotoxemia and also attenuates local inflammatory responses. J. Exp. Med. 181: 2289-2293.

4. Li P, Allen H, Banerjee $S$, et al. (1995) Mice deficient in IL-1 $\beta$-converting enzyme are defective in production of mature IL- $1 \beta$ and resistant to endotoxic shock. Cell 80: 401-411.

5. McIntyre KW, Stepan GJ, Kolinsky KD, et al. (1991) Inhibition of interleukin 1 (IL-1) binding and bioactivity in vitro and modulation of acute inflammation in vivo by IL-1 receptor antagonist and anti-IL-1 receptor monoclonal antibody. $J$. Exp. Med. 173: 931-939.

6. Cross AS, Opal S. (1994) Therapeutic intervention in sepsis with antibody to endotoxin: is there a future? J. Endotox. Res. 1: 57-69.

7. Fisher CJ, Agosti JM, Opal SM, et al. (1996) Treatment of septic shock with the tumor necrosis factor receptor:Fc fusion protein. N. Engl. J. Med. 334: 1697-1701.

8. Opal SM, Fisher CJ, Dhainaut JF, et al. (1997) Confirmatory interleukin-1 receptor antagonist trial in severe sepsis: a phase III, randomized, dou- ble-blind, placebo-controlled, multicenter trial. Crit. Care Med. 25: 1115-1124.

9. Freudenberg MA, Keppler D, Galanos C. (1986) Requirement for lipopolysaccharide-responsive macrophages in galactosamine-induced sensitization to endotoxin. Infect. Immun. 51: 891-895.

10. Pittet D, Rangel-Frausto S, Li N, et al. (1995) Systemic inflammatory response syndrome, sepsis, severe sepsis and septic shock: incidence, morbidities and outcomes in surgical ICU patients. Intensive Care Med. 21: 302-309.

11. Liang P, Pardee AB. (1992) Differential display of eukaryotic messenger RNA by means of the polymerase chain reaction. Science 257: 967-971.

12. Wiegand G, Selleng $K$, Gründling $M$, Jack RS. (1999) Isolation of highly enriched monocytes from $10 \mathrm{ml}$ samples of heparinised whole blood. Clin. Chem. Lab. Med. (in press).

13. van Rhenen DJ, Jalink W, Huijgens PC. (1983) Esterase staining in monocytes. J. Clin. Pathol. 36: 236-237.

14. Überla $K$, Platzer C, Diamantstein T, Blankenstein T. (1991) Generation of competitor DNA fragments for quantitative PCR. PCR Methods Appl. 1: 136-139.

15. Bauer D, Müller H, Reich J, et al. (1994) Identification of differentially expressed mRNA species by an improved display technique (DDRT-PCR). Nucl. Acids Res. 21: 4272-4280.

16. Welford SM, Greg J, Chen E, et al. (1998) Detection of differentially expressed genes in primary tumor tissues using representational difference analysis coupled to microarray hybridisation. $\mathrm{Nucl}$. Acids Res. 26: 3059-3065. 